\title{
Sensitivity Limits for Near- Infrared Gas Sensing with Suspended-core PCFs directly coupled with VCSELs
}

\author{
J. Chen ${ }^{1,2}$, A. Hangauer ${ }^{1,2}$, R. Strzoda ${ }^{1}$, T. G. Euser ${ }^{3}$, J. S. Y. Chen ${ }^{3}$, \\ M. Scharrer ${ }^{3}$, P. St. J. Russell ${ }^{3}$ and M.-C. Amann ${ }^{2}$ \\ 1. Corporate Research and Technology, Siemens AG, Otto-Hahn-Ring 6, D-80200 München \\ 2. Walter Schottky Institut, Technische Universität München, Am Coulombwall 3, D-85748 Garching \\ 3. Max Planck Institute for the Science of Light, Günther-Scharowsky-Str. 1, D-91058 Erlangen \\ E-mail :jia.chen@wsi.tum.de
}

\begin{abstract}
We demonstrate direct-coupling of vertical-cavity surface-emitting lasers to suspended-core PCFs and present characterization of this gas sensor. The spectral background and power-overlap from $763-2004 \mathrm{~nm}$ are measured to determine the quantitative detection sensitivity.

(C) 2010 Optical Society of America

OCIS Codes: 060.5295 (Photonic crystal fibers), 140.7260 (Vertical cavity surface emitting lasers), 300.6260 (Spectroscopy, diode lasers)
\end{abstract}

\section{Introduction}

The small divergence of vertical-cavity surface-emitting laser (VCSEL) emission (FWHM: 10-20 degree) makes them attractive sources for direct integration with silica suspended-core photonic crystal fibers (PCFs). The light propagating in the solid core of the index-guiding PCF probes the sample via its evanescent field. The fiber provides much longer path length and greatly reduced sample volume requirement of $0.7 \mu \mathrm{L} / \mathrm{m}$ compared to conventional sample cells. A fiber sensor is also ideal for distributed gas sensing if suitable gas inlets along the fiber length are implemented, allowing for gas measurement in situations where it is difficult to use an open pass configuration. Recently, it was shown that suspended-core PCFs can be used for quantitative gas- and liquid sensing [1-3], but so far only high absorption has been detected (the absorption is more than $10 \%$ ).

In this paper, we present quantitative measurements of the spectral background of the transmission function using VCSELs that are directly coupled to suspended-core PCF. The spectral background usually limits the sensitivity of gas detection. Gas sensing experiments probing different wavelengths were carried out, which also allowed for reliable measurements of the overlap between gas-filled cladding and light, i.e., the wavelength dependence of the overlap between the evanescent field and the gas.

\section{Experimental results}

Light emitted by the VCSELs was launched directly to the core of the PCF, placed at $<10 \mu \mathrm{m}$ distance from the VCSEL. The typical launch efficiencies were $13 \%(763 \mathrm{~nm})$ and $15 \%(1674 \mathrm{~nm})$, which were in good agreement with surface area ratio between VCSEL and fibre core. The mode intensity profile at the fiber output after a $2 \mathrm{~m}$ length of fiber was imaged with a CCD camera to confirm that the light propagates in the solid core of the fiber (see Fig. 1). By comparing the beam profiles at $1674 \mathrm{~nm}$ and $2004 \mathrm{~nm}$, the overlap between the core mode and air holes was shown to increase with wavelength.

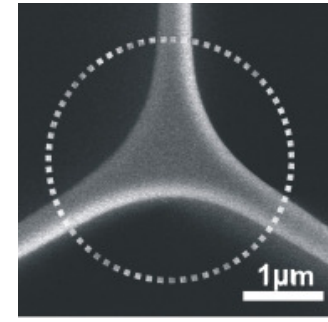

(a)

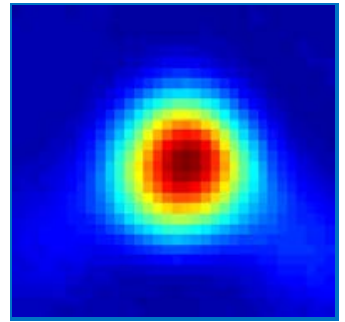

(b)

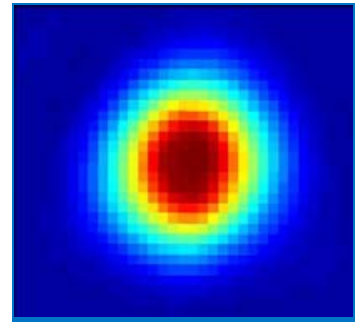

(c)

Fig. 1 (a) SEM of the air-suspended solid-core PCF with core diameter of $1 \mu \mathrm{m}$. The dashed circle indicates the approximate size of the directly coupled VCSEL with aperture size of $5 \mu \mathrm{m}$. (b) Measured mode intensity profile at the output of the fiber for $1674 \mathrm{~nm}$ and (c) for $2004 \mathrm{~nm}$, recorded by a CCD camera. The scale of (b) and (c) is roughly equal to the scale of SEM plot. 
(a)

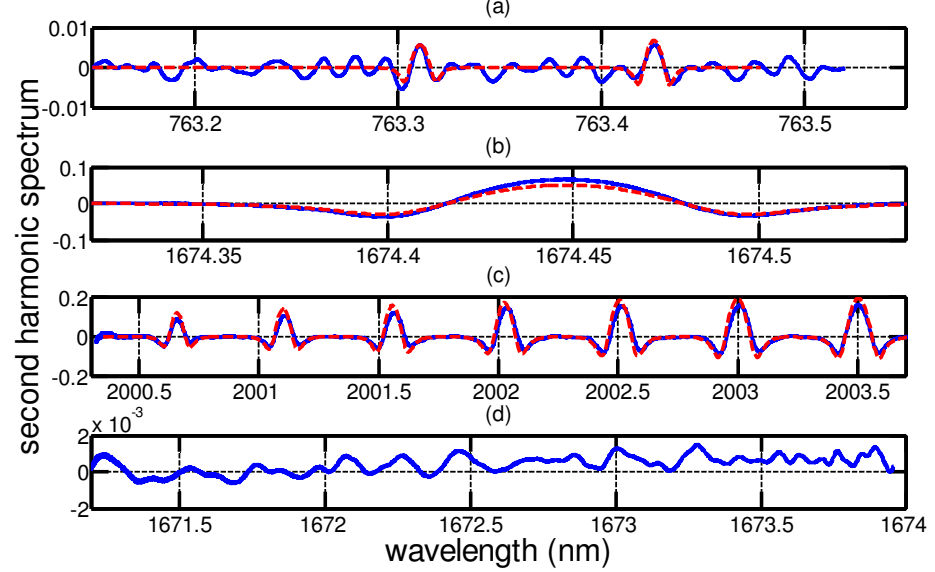

(e)

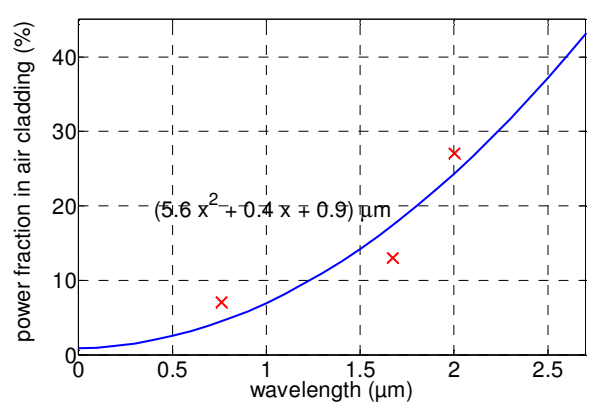

Fig. 2 Second harmonic spectra normalized to the DC signal for different gases/wavelengths: (a) $100 \% \mathrm{O}_{2}$ and $763 \mathrm{~nm}$; (b) $10 \% \mathrm{CH}$ $1674 \mathrm{~nm}$; (c) $10 \% \mathrm{CO}_{2}$ and $2004 \mathrm{~nm}$; (d) background spectrum - for a nitrogen filled fiber using a $1674 \mathrm{~nm}$ VCSEL. Blue solid curves represent experimental data and red dashed curves the corresponding curve fit with HITRAN data. (e) Power overlap determined by comparing the measured and theoretical absorption linestrength. The blue curve is a second order polynomial fit including the origin $(0,0)$.

In-fiber near infrared gas sensing was performed with VCSELs at wavelengths of $763 \mathrm{~nm}, 1674 \mathrm{~nm}$ and $2004 \mathrm{~nm}$, near the absorption lines of $\mathrm{O}_{2}, \mathrm{CH}_{4}$ and $\mathrm{CO}_{2}$, respectively. The wavelength was tuned via the laser current, and the resulting normalized second harmonic spectra [4] are shown in Fig. 2. The power overlap between light and gas, $\Phi$, was obtained by comparing the measured and theoretical (HITRAN) line strength [5] and determined to be $7 \%(763 \mathrm{~nm}), 13 \%(1674 \mathrm{~nm}), 27 \%(2004 \mathrm{~nm})$, increasing with wavelength as expected (Fig. 2e). Since the exact gas concentration and the length of the fiber were known, the resulting error is mostly due to the inaccuracy of the HITRAN data, which is typically less than $10 \%$.

The spectral background was found to have a peak-to-peak amplitude of less than $2 \times 10^{-3}$ (Fig. 2 (d)) at all three wavelengths. A fine structure of ripples is observed in the spectral background. We exclude that this structure is caused by absorption losses, since they would typically result in more broadband features. One possibility for the observation is a tiny fraction of light is unintentionally coupled and expected to propagate in the outer jacket of the fibre, which acts as a multimode waveguide. As we use a large photodetector at the end of the fibre, both light from the core mode and the jacket modes are collected. The loss coefficients or backscattering coefficients for each mode can have a different wavelength dependence, which would result in wavelength selective transmission, as observed in the experiment.

\section{Conclusion}

In this paper, the spectral background of suspended-core PCF was characterized for the first time to determine the detection sensitivity of the fiber sensor, which was of the order of $10^{-3}$. By comparing the theoretical and measured absorption line strengths for different gases, the power overlap as a function of wavelength was also obtained. The robust integration of VCSEL light sources and photonic crystal fibers by direct coupling is an important step towards compact gas sensors. Future work includes the investigation and further reduction of the spectral background for instance by avoiding the jacket modes.

The authors gratefully acknowledge the financial support by the Federal Ministry of Education and Research of Germany (Project 'NOSE', No. 13N8772) and VERILAS GmbH, Garching for providing VCSELs at $1670 \mathrm{~nm}$ and $2004 \mathrm{~nm}$.

\section{References}

[1] A. S. Webb et al., "Suspended-core holey fiber for evanescent-field sensing," Opt. Eng. 46, 010503 ( 2007).

[2] C. M. Cordeiro et al., "Single-design-parameter microstructured optical fiber for chromatic dispersion tailoring and evanescent field enhancement" Opt. Lett. 32, 3324 (2007).

[3] T. G. Euser et al., "Quantitative broadband chemical sensing in air-suspended solid-core fibers," J. Appl. Phys. 103, 103108 (2008).

[4] J. Reid and D. Labrie, "Second-harmonic detection with tunable diode lasers - comparison of experiment and theory," Appl. Phys. B 26, 203 (1981).

[5] L. Rothman, “The HITRAN 2004 molecular spectroscopic database,” J. Quant. Spectrosc. Radiat. Transfer 96, 139 (2005). 